EESTI NSV TEADUSTE AKADEEMIA TOIMETISED. 20. KÖIDE KEEMIA * GEOLOOGIA. 1971, NR. 3

ИЗВЕСТИЯ АКАДЕМИИ НАУК ЭСТОНСКОИ ССР. ТОМ 20 ХИМИЯ * ГЕОЛОГИя. 1971, № 3

УдК 541.12.034.6

В. ЖАРОВ, ТАТЬЯНА ВИТМАН, ХЕЛЛЕ ВИИТ, Л. КУДРЯВЦЕВА

\title{
ИЗОТЕРМИЧЕСКОЕ ОПРЕДЕЛЕНИЕ ДАВЛЕНИЯ ПАРА НАД ЖИДКОСТЬЮ
}

В настоящем сообщении изложен метод определения давления пара над жидкостью при постоянной температуре, разработанный для малых количеств исходных веществ и смесей.

\section{Теоретическая часть}

Внешнее давление на жидкость в приборе (см. рисунок) уравновешивается в капилляре следующим образом:

$$
P=P_{\mathrm{c \tau}}+P_{0}+P_{\mathrm{B}}
$$

где

$$
P \text { - давление в системе, }
$$

$P_{\text {ст }}=\frac{d_{\text {ж }}}{d_{\mathrm{Hg}}} \cdot h-$ давление столба жидкости в капилляре, равное произведению отношения плотностей жидкости и ртути на высоту столба жидкости,

$P_{0}$ - давление насыщенного пара при данной температуре,

$P_{\text {в }}$ - парциальное давление воздуха, оставшегося в капилляре.

Прибор калибруется по жидкости, давление пара которой при данной температуре известно.

Для двух уровней указанной жидкости в капилляре можно записать два уравнения типа (1):

$$
P_{\mathrm{B}}^{1}=P^{1}-P_{\mathrm{cr}}^{1}-P_{0} \text { и } P_{\mathrm{B}}^{2}=P^{2}-P_{\mathrm{c \tau}}^{2}-P_{0},
$$

в которых индексами 1 и 2 обозначены величины, определенные для первого и второго уровня столба жидкосги в капилляре. Из уравнений (2) можно рассчитать значения $P_{\mathrm{B}}^{i}$. Исключив из (2) $P_{0}$, получим

$$
P_{\mathrm{B}}^{2}-F_{\mathrm{B}}^{1}=\Delta P_{\mathrm{B}}=\left(P^{2}-P^{1}\right)-\left(P_{\mathrm{cT}}^{2}-P_{\mathrm{cT}}^{1}\right) \text {. }
$$

С другой стороны, считая воздух идеальным газом, можем записать для двух уровней в капилляре

$$
P_{\mathrm{B}}^{1} \cdot V^{1}=P_{\mathrm{B}}^{2} \cdot V^{2},
$$

где $V^{1}$ и $V^{2}$ объемы, занимаемые воздухом и насыщенным паром (отсекаемые столо́ом жндкости в капилляре) на каждом из двух уровней. Отсюда.

$$
P_{\mathrm{B}}^{1}=P_{\mathrm{B}}^{2} \cdot \frac{V^{2}}{V^{1}} \quad \text { и } \quad P_{\mathrm{B}}^{2}=P_{\mathrm{B}}^{1} \cdot \frac{V^{1}}{V^{2}}
$$


с учетом которых из левой части уравнения (3) получим

$$
\frac{V^{1}}{V^{2}}=\frac{\Delta P_{\mathrm{E}}}{P_{\mathrm{B}}^{1}}+1 \quad \text { и } \quad \frac{V^{2}}{V^{1}}=1-\frac{\Delta P_{\mathrm{n}}}{P_{\mathrm{B}}^{2}} .
$$

Отношения объемов, постоянные для данных уровней столбов жидкости в капилляре, используются в дальнейших исследованиях, при которых давление в системе и давление столба жидкости определяются именно для этих двух уровней. Искомая величина $P_{0}$ рассчитывается при этом из уравнений

$$
P_{0}=P^{\mathrm{i}}-P_{\text {ст }}^{1}-P_{\mathrm{B}}^{1} \text { или } P_{0}=P^{2}-P_{\mathrm{cт}}^{2}-P_{\mathrm{B}}^{2}
$$

с учетом того, что $P_{\mathrm{B}}^{1}=\frac{\Delta P_{\mathrm{n}}}{\frac{V^{1}}{V^{2}}-1}$ и $P_{\mathrm{B}}^{2}=\frac{\Delta P_{\mathrm{n}}}{1-\frac{V^{2}}{V^{1}}}$.

При этом $P^{i}$ и $P_{\mathrm{cr}}^{i}$ определяются из опыта, $\Delta P_{\text {в }}$ рассчитывается с помощью уравнения (3), .а отношения объемов определены при калибровке лрибора.

\section{Экспериментальная часть}

$\mathrm{Ha}$ рисунке изображен прибор для измерения давления пара, отвод 4 которого соединен с системой измерения и автоматического регулирования давления. Прибор помещен в стеклянную трубку, по которой циркулирует жидкость из термостата. Температура в приборе контролируется термометром 5. Через отвод 3 в сосуд наливается исследуемая жидкость $(\sim 2$ мл). Путем откачки из капилляра удаляется часть воздуха, место которого при увеличении давления заннмает жидкость. При данной температуре и атмосферном давлении в капилляре оставляется такое количество воздуха, чтобы уровень жидкости в нем был несколько выше верхней отметки. Затем путем откачки уровень жидкости устанавливается последовательно у обеих отметок, и после достижения равновесия (20 мин) измеряется давление в системе и высота столба жидкости в капилляре. Для чистого вещества последовательность установления уровня жидкости у обеих отметок не имеет значения, для смеси же целесообразнее устанавливать уровень первоначально у нижней отметки, а затем, повышая давление в системе, -. у верхней отметки. Таким путем удается избежать лишней откачки н уменьшить изменение состава смеси.

В настоящей работе прибор был прокалиброван по бензолу и циклогексану при температурах 30, 40. 50 С. Давление в системе измерялось с помощью манометра МЧР-3 с точностью $\pm 0,1$ мм рт. ст. Высота столба жидкости в капилляре определялась катетометром КМ-6. Для поддержания в системе постоянного давления применялся картезиан-

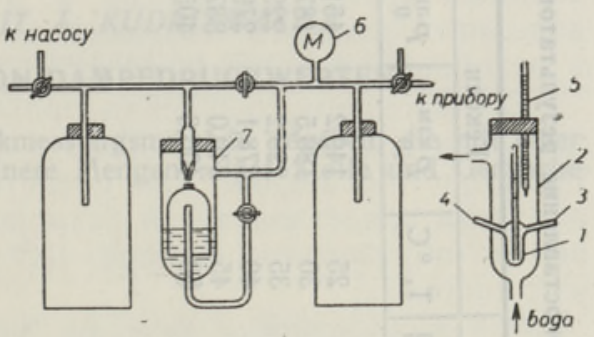

Схема установки для изотермического определения давления пара над жидкостью.

1 - Прибор для измерения давлення пара, 2 - стеклянная трубка для термостатирующей жидкости, 3,4- отводы, 5 - термометр, 6 - манометр, 7 - маностат. ский маностат 7 (см. рисунок).

В табл. 1 и 2 результаты измерения давления паров чистых веществ и бинарных смесей соответственно сравниваются с данными, приведенными в литературе. Состав смесей, изготовляемых по весу, анализиро- 
ำ

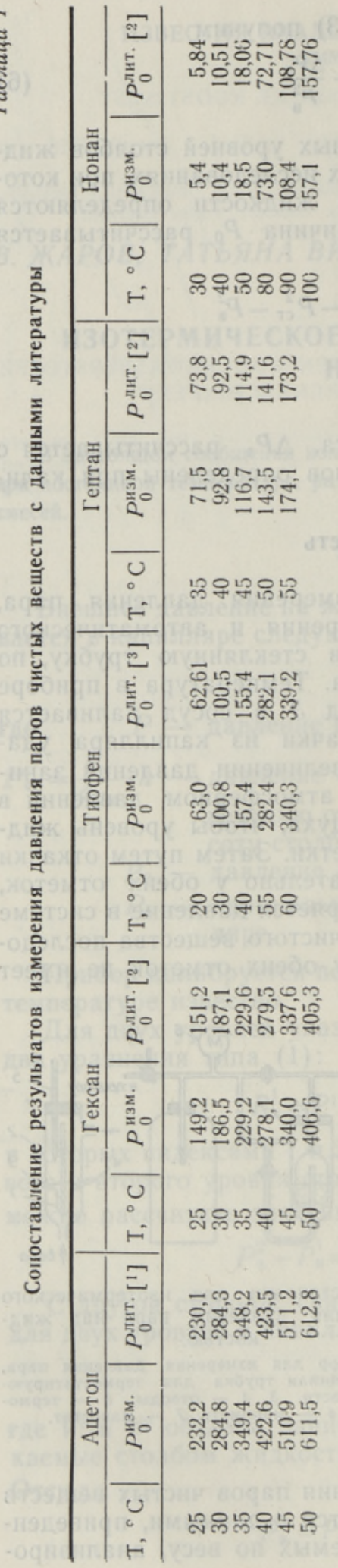

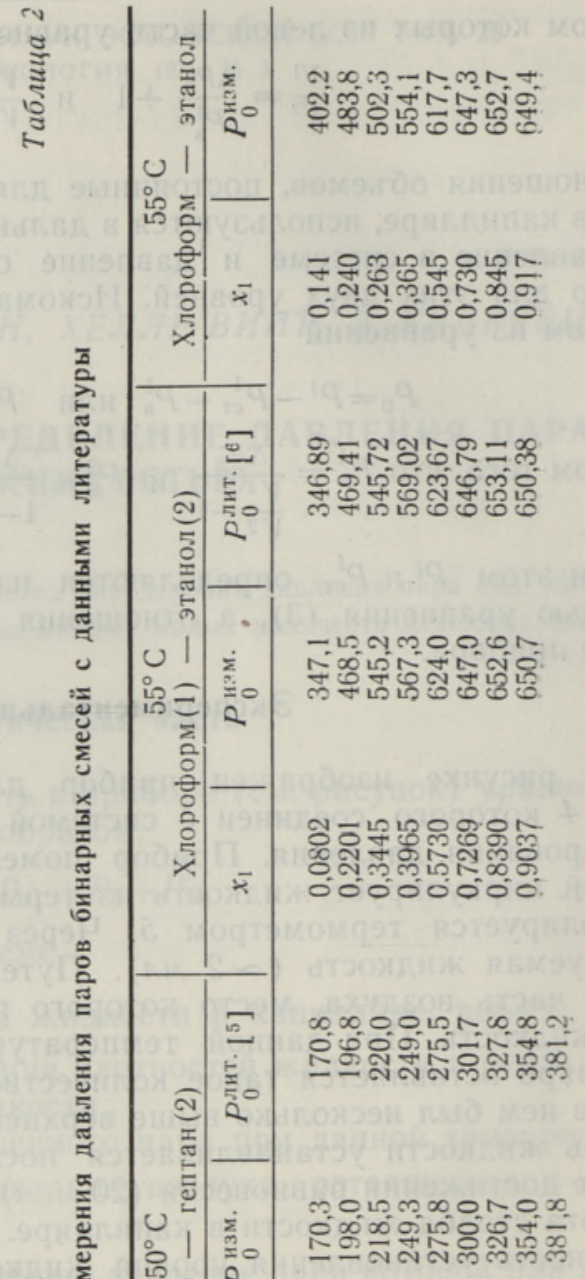

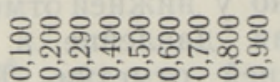

L $N$ ᄂ

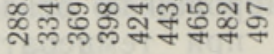

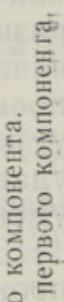

$0, \pi$.

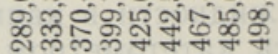

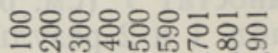
00000000 
вался после окончания опыта. Нанбольшее изменение в составе (до2 мол. \%) наблюдалось в системе хлороформ - этанол. Значения $P_{0}^{\text {нзм }}$ отнесены к конечным концентрациям. Необходимые для сравнения значения $P_{0}^{\text {нзм. }}$, соответствующие концентрациям, указанным в работе $\left[{ }^{3}\right]$, были получены путем интерполяции из кривой зависимости $P_{0}=f\left(x_{1}\right)$, построенной по данным, полученным из опыта и приведенным в двух последних столбцах табл. 2.

Из таблиц следует, что расхождение опытных данных с литературными не превышает \pm 2 мм рт. ст.

\section{Л И ТЕ Р А Т Р А}

1. Holmes M. J., van Winkle M., Ind. Engng Chem., 62, Nr. 1, 21 A (1970).

2. Физико-химические свойства индивидуальных углеводородов. М., 1960.

3. Вей сбергер А., Проскауэр Э., Риддик Дж., Ту п С., Органические растворители. М.-Л., 1955.

4. B rown I., S m it h F., Aust. J. Chem., 10, Nr. 4, 423 (1957).

5. S m ith C. P., Eng el E. W., J. Am. Chem. Soc., 51, Nr. 9, 2646 (1929).

6. S c a tch ard G., R a y mond C. L., J. Am. Chem. Soc., 60, 1278 (1938).
Институт химии
Академии наук Эстонской ССР
Поступила в редакшию:
20/XI 1970

\section{ZAROV, TATJANA VITMAN, HELLE VIIT, L. KUDRJAVTSEVA}

\section{AURURŌHU ISOTERMILINE MAARAMINE}

Esitatakse väikeste hulkade puhaste ainete ja segude staatiline aururōhu määramise: meetod, mis võimaldab aururőhku määrata täpsusega \pm 2 torri.

W. ZAROW, TATJANA WITMAN, HELLE WIIT, L. KUDRJAWZEWA

\section{ISOTHERMISCHE MESSUNG VON DAMPFDRUCKWERTEN}

Es wird eine schnelle statische Dampfdruckmessungsmethode gegeben, die mit einer: Genauigkeit von \pm 2 Torr arbeitet und für kleinere Mengen reiner Stoffe und Gemische. anwendbar ist. 